\title{
Clinical and microbiological characteristics of nosocomial, healthcare-associated, and community-acquired Klebsiella pneumoniae infections in Guangzhou, China
}

\author{
Tingting Le ${ }^{1,2+}$, Ling Wang ${ }^{1 \dagger}$, Chaoying Zeng ${ }^{1}$, Leiwen $\mathrm{Fu}^{3}$, Zhihua Liu ${ }^{4}$ and Jing Hu ${ }^{1 *}$
}

\begin{abstract}
Background: Klebsiella pneumoniae (K. pneumoniae) is a common pathogen associated with hospital and community-onset infections. This study aimed to compare the clinical and microbiological characteristics of nosocomial, healthcare-associated (HCA), and community-acquired (CA) K. pneumoniae infections.

Methods: Clinical data were extracted from electronic medical records and analyzed retrospectively. Antimicrobial susceptibility and extended-spectrum beta-lactamase (ESBL) production were determined for all identified strains. Carbapenemase and ESBL genes were amplified by PCR. Genotyping of carbapenem-resistant K. pneumoniae (CRKP) and ESBL-producing strains was performed by pulsed-field gel electrophoresis (PFGE).

Results: Of 379 K. pneumoniae infections, 98 (25.9\%) were nosocomial, 195 (51.5\%) were healthcare-associated, and 86 (22.6\%) were community-acquired. Hematological malignancy ( $\mathrm{OR}=4.467)$, and hypertension $(\mathrm{OR}=2.08)$ and cerebral vascular disease $(O R=2.486)$ were associated with nosocomial and HCA infections respectively, when compared to CA infections. Overall, the incidence of antimicrobial resistance for the majority of agents tested was similar between nosocomial and HCA infections $(P>0.05)$ and both groups had a higher incidence than CA infections $(P<0.05)$. Moreover, $95.1 \%$ (78/82) of CRKP strains were isolated from the nosocomial and HCA groups. The bla $a_{\text {KPC }}$ was the most prevalent carbapenemase gene among CRKP strains $(80.5 \%, 66 / 82)$. ESBL-producing strains were prevalent among nosocomial (40.8\%), HCA (35.9\%) and CA groups (24.4\%). The bla $a_{C T X-M-9-\text { group }}$ and bla $a_{C T X-M-1 \text {-group }}$ genes were predominant in nosocomial (65.0\%) and CA strains (66.7\%), respectively. PFGE results showed ESBL-producing and CRKP strains were genetically diverse. Identical PFGE profiles were observed among HCA and nosocomial strains.

Conclusions: Nosocomial and HCA K. pneumoniae infections presented similar clinical features and antimicrobial resistance, and both two types of infections were different to CA infections. CRKP and ESBL-producing strains were disseminated mainly in HCA and nosocomial groups, and showed a clonal diversity. The cross transmission of CRKP was existed among HCA and nosocomial patients. This finding suggests that similar empirical therapy should be considered for patients with nosocomial and HCA K. pneumoniae infections and bacterial resistance surveillance of these infections is necessary.
\end{abstract}

\footnotetext{
*Correspondence: hjalzh@smu.edu.cn

${ }^{\dagger}$ Tingting Le and Ling Wang have contributed equally

1 Department of Nosocomial Infection Administration, Zhujiang Hospital,

Southern Medical University, Guangzhou 510282, Guangdong, China

Full list of author information is available at the end of the article
}

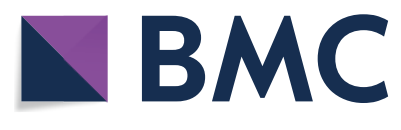

(c) The Author(s) 2021. Open Access This article is licensed under a Creative Commons Attribution 4.0 International License, which permits use, sharing, adaptation, distribution and reproduction in any medium or format, as long as you give appropriate credit to the original author(s) and the source, provide a link to the Creative Commons licence, and indicate if changes were made. The images or other third party material in this article are included in the article's Creative Commons licence, unless indicated otherwise in a credit line to the material. If material is not included in the article's Creative Commons licence and your intended use is not permitted by statutory regulation or exceeds the permitted use, you will need to obtain permission directly from the copyright holder. To view a copy of this licence, visit http://creativecommons.org/licenses/by/4.0/. The Creative Commons Public Domain Dedication waiver (http://creativeco mmons.org/publicdomain/zero/1.0/) applies to the data made available in this article, unless otherwise stated in a credit line to the data. 
Keywords: Klebsiella pneumoniae infections, Nosocomial, Healthcare-associated, Community-acquired, Antimicrobial resistance

\section{Background}

Klebsiella pneumoniae is a threatening human pathogen that causes various diseases, including respiratory tract and urinary tract infections, blood infections, and liver abscesses [1]. Traditionally, bacterial infections have been classified into community-acquired and nosocomial infections according to the route of infection. As the healthcare-associated effects on community-onset infections cannot be overlooked, these infections have been divided into community-onset healthcare-associated (HCA) infections for patients with a recent history of medical care, and communityacquired infections for patients without [2]. Previous studies have described that HCA infections are different from CA infections and share some similarities with nosocomial infections regarding clinical characteristics, outcome and microbiological features $[2,3]$. Although there have been studies on HCA infections, the data about antimicrobial resistance and molecular features of HCA K. pneumoniae infections are limited $[4,5]$. Inadequate understanding of these infections could lead to inappropriate empirical treatment and, in consequence, higher morbidity and mortality.

Antimicrobial resistance in $K$. pneumoniae is a significant concern to public health. The CRKP and ESBLpositive $K$. pneumoniae strains are the most important and challenging drug-resistant bacteria, leading to limited treatment options and high mortality [6-8]. Thus, understanding the distribution of such highly resistant strains in both hospitals and communities is vital to planning interventions.

The production of carbapenemase is the main carbapenem-resistance mechanism in K. pneumoniae [9]. Carbapenemase encoding genes reported worldwide mainly include $b l a_{\mathrm{KPC}}, b l a_{\mathrm{NDM}}$, and $b l a_{\mathrm{OXA}-48 \text {-like }}[10-$ 12]. Bla $a_{\mathrm{SHV}}, b l a_{\mathrm{TEM}}, b l a_{\mathrm{CTX}-\mathrm{M}}$, and $b l a_{\mathrm{OXA}}$ are the major ESBL genes identified to date $[13,14]$. These antibiotic resistance genes are normally located on mobile genetic elements, such as plasmids and transposons, thus, they can be widely spread in both nosocomial and community environments $[15,16]$.

Strain typing is of great significance for epidemiological surveillance. PFGE is a gold standard for the genotyping of strains. The molecular typing of $K$. pneumoniae isolates contributes to the identification of specific clone dissemination and the source of infections, on which the prevention approach depends.
Currently, there are limited data comparing the clinical and microbiological characteristics of nosocomial, $\mathrm{HCA}$, and CA K. pneumoniae infections. And the study data on antimicrobial resistance of HCA K. pneumoniae infections is scarce. Furthermore, strains isolated from different regions present their own distinct clinical and microbiological features. Therefore, this study aimed to characterize the clinical features, antimicrobial resistance and the distribution of CRKP and ESBL-positive strains among nosocomial, HCA, and CA infections caused by K. pneumoniae, and to characterize the genetic relatedness of CRKP and ESBL-positive isolates identified from the three different groups. The results from this study will be helpful in developing effective preventive and treatment strategies against $K$. pneumoniae infections both in hospitals and in communities.

\section{Methods}

\section{Study design and population}

This retrospective study was conducted at a 2300-bed tertiary hospital in Guangzhou, China. All patients presenting K. pneumoniae infections from January 2019 to December 2019 were included in the study. K. pneumoniae strains were consecutively collected from these patients. For patients with multiple $K$. pneumoniae isolates, only the first isolate was analysed. During the study period, the number of admittances and outpatient treatments were about 103,630 and 1,610,618, respectively.

The definitions of nosocomial, HCA, and CA K. pneumoniae infection was based on previously described criteria [2]. A nosocomial $K$. pneumoniae infection was defined as an infection that occurred $48 \mathrm{~h}$ after the patient's admission. A HCA K. pneumoniae infection was defined as an infection developing within $48 \mathrm{~h}$ of admission in patients presenting any of the following risk factors [2]: a history of intravenous therapy or renal dialysis in the 30 days before the K. pneumoniae infection; a history of hospitalization for 2 or more days in the three months before the $K$. pneumoniae infection; or residence in a nursing home or long-term care facility. A CA $K$. pneumoniae infection was defined as an infection occurring in patients who did not fulfill the definitions of nosocomial or HCA infections.

\section{Clinical data collection}

We extracted the following clinical data from electronic records for all patients: demographic characteristics, underlying disease, site of infection, immunosuppression, 
and mortality. Exposure to prior antimicrobial treatment was defined as any treatment received for at least $48 \mathrm{~h}$ in the 30 days before the K. pneumoniae infection.

\section{Bacterial identification and antimicrobial susceptibility testing}

A total of $379 \mathrm{~K}$. pneumoniae strains were collected from patients during the study period. The Vitek 2 system (bioMérieux, France) was used for bacterial identification. Antimicrobial susceptibility and the ESBL phenotype were determined using the Vitek 2 System following the Clinical and Laboratory Standards Institute guidelines (CLSI, 2019). The antibiotics tested included ampicillin (AMP), piperacillin/tazobactam (TZP), amoxicillin/ clavulanic acid (AMC), cefoperazone/sulbactam (CPS), cefazolin (CZO), cefuroxime (CXM), cefepime (FEP), ceftriaxone (CRO), ciprofloxacin (CIP), cefoxitin (FOX), ceftazidime (CAZ), imipenem (IMP), amikacin (AMK), gentamicin (GEN), ertapenem (ERP), meropenem (MEM), aztreonam (ATM), levofloxacin (LVE), trimethoprim/sulfamethoxazole (SXT), and tigecycline (TGC). The breakpoint of tigecycline was based on the standard of the United States Food and Drug Administration (FDA). Escherichia coli ATCC 25,922 and K. pneumoniae ATCC 700,603 served as quality control strains.

Multidrug resistant (MDR) strains were defined as strains with no susceptibility to at least three different classes of antimicrobials [17]. Carbapenem-resistant $K$. pneumoniae (CRKP) isolates were defined as isolates that displayed resistance to one or more carbapenem agents such as meropenem, ertapenem, or imipenem [18].

\section{Detection of resistance genes}

Polymerase chain reaction (PCR) was performed to detect carbapenemase genes (bla $a_{\mathrm{KPO}}, b l a_{\mathrm{NDM}}, b l a_{\mathrm{GIM}}$, bla SPM $_{1}, b l a_{\mathrm{IMP}}, b l a_{\mathrm{SIM}}, b l a_{\mathrm{VIM}}, b l a_{\mathrm{IMI}}$ and $\left.b l a_{\mathrm{OXA}-48-\text { like }}\right)$ in the CRKP strains and ESBLs genes $\left(b l a_{\mathrm{TEM}}, b l a_{\mathrm{SHV}}\right.$, bla $a_{\mathrm{CTX}-\mathrm{M} \text {-group }} \quad b l a_{\mathrm{OXA}-1 \text {-like, }} \quad b l a_{\mathrm{CTX}-\mathrm{M}-1 \text {-group, }} \quad b l a_{\mathrm{CTX}-\mathrm{M}-2-}$ group, bla $a_{\mathrm{CTX}-\mathrm{M} \text {-9-group }}$ ) in the ESBL-producing strains [17, 19-22]. The commercial kits Green Taq Mix (Vazyme, Nanjing, China) and specific primers were used to detect resistance genes. Amplification conditions are as follows: initial denaturation at $95^{\circ} \mathrm{C}$ for $3 \mathrm{~min}, 30$ cycles of $95^{\circ} \mathrm{C}$ for $40 \mathrm{~s}$, primer annealing temperature for $40 \mathrm{~s}, 72^{\circ} \mathrm{C}$ for $50 \mathrm{~s}$, and $72^{\circ} \mathrm{C}$ for $7 \mathrm{~min}$. Bacterial strains with resistance genes detected by PCR and DNA sequencing were used as positive controls for the subsequent PCR runs. The distilled water was used as a negative control. Amplification products were analyzed by agarose gel electrophoresis. The specific primers are presented in Additional file 1: Table S1. The PCR products were sequenced and the sequencing results were analyzed on the BLAST website (http://blast.ncbi.nlm.nih.gov).

\section{Pulsed-field gel electrophoresis (PFGE)}

All 186 CRKP or ESBL-producing strains were subjected to PFGE following the PulseNet standardized procedure (http://www.cdc.gov/pulsenet/protocols.htm). Briefly, the strain DNA were digested with Xbal for $2 \mathrm{~h}$ at $37^{\circ} \mathrm{C}$. Electrophoresis was performed using the CHEFDRIII system (120 angle, $6 \mathrm{~V} / \mathrm{cm})$, with a running time of $19 \mathrm{~h}$ and a switch time of 2.16-54 s. The PFGE fingerprint patterns were uploaded to the $K$. pneumoniae database of the Chinese Pathogen Identification Net (http://139.9.117.189/CPIN/). A Dice coefficient-based PFGE dendrogram was constructed using the unweighted pair-group mean Analysis. Isolates were grouped into the same PFGE cluster if they shared $\geq 80 \%$ similarity.

\section{Statistical analyses}

Categorical variables were analyzed by Chi-square or Fisher's exact tests (SPSS software, version 20.0). P-values $<0.05$ (two-tailed) were considered statistically significant. Multivariable logistic regression analysis was conducted to identify variables associated with nosocomial, HCA, and CA infections. Variables regarding age, underlying disease, and immunosuppression with $P<0.05$ in the univariate analysis were included in the multivariable logistic regression model.

\section{Results \\ Clinical features of $K$. pneumoniae infection}

In total, 379 patients with $K$. pneumoniae infection were included (98 nosocomial, $195 \mathrm{HCA}$ and $86 \mathrm{CA}$ infections) in the analysis. The incidence of $K$. pneumoniae infections was approximately 3.66 per 1000 admissions in the hospital. Male patients accounted for $64.12 \%$ of $K$. pneumoniae infections. The overall 30-day crude mortality rate was $12.4 \%(47 / 379)$.

The clinical features of $K$. pneumoniae infections are presented in Table 1 . When compared to HCA (6.2\%) and CA infections (5.8\%), nosocomial infections (21.4\%) occurred more frequently in patients aged $\leq 1$ years old. Multivariate analysis further showed that patients aged $\leq 1$ years old (nosocomial vs. $\mathrm{HCA}$ : $\mathrm{OR}=5.665$, nosocomial vs. $\mathrm{CA}$ : $\mathrm{OR}=4.456)$ was an independent variable associated with nosocomial infections (Table 2).

There were differences between the three groups regarding the infection type, with bloodstream infections being dominant in nosocomial patients (45.9\%), respiratory tract infections being frequent in HCA patients (33.9\%), and skin and soft tissue infections being predominant in CA patients (25.6\%).

Nosocomial infections and HCA infections shared similar features in terms of underlying diseases. However, $\mathrm{CA}$ infections differed from both nosocomial and $\mathrm{HCA}$ 
Table 1 Clinical features of $K$. pneumoniae infections

\begin{tabular}{|c|c|c|c|c|c|c|}
\hline \multirow[t]{2}{*}{ Variable } & \multirow{2}{*}{$\begin{array}{l}\text { Nosocomial } \\
(n=98)\end{array}$} & \multirow{2}{*}{$\begin{array}{l}\text { HCA } \\
(n=195)\end{array}$} & \multirow{2}{*}{$\begin{array}{l}\text { CA } \\
(n=86)\end{array}$} & \multicolumn{3}{|l|}{$P$-value } \\
\hline & & & & $\begin{array}{l}\text { HCA } \\
\text { versus Nosocomial }\end{array}$ & $\begin{array}{l}\text { CA } \\
\text { versus Nosocomial }\end{array}$ & CA versus $\mathrm{HCA}$ \\
\hline \multicolumn{7}{|l|}{ Age (years) } \\
\hline$\leq 1$ & $21(21.4 \%)$ & $12(6.2 \%)$ & $5(5.8 \%)$ & 0.000 & 0.002 & 0.912 \\
\hline $2-10$ & $10(10.2 \%)$ & $5(2.6 \%)$ & $1(1.2 \%)$ & 0.005 & 0.010 & 0.671 \\
\hline $11-18$ & $8(8.2 \%)$ & $1(0.5 \%)$ & $2(2.3 \%)$ & 0.001 & 0.107 & 0.223 \\
\hline $19-59$ & $34(34.7 \%)$ & 89 (45.6\%) & $48(55.8 \%)$ & 0.073 & 0.004 & 0.116 \\
\hline$\geq 60$ & $25(25.5 \%)$ & $88(45.1 \%)$ & $30(34.9 \%)$ & 0.001 & 0.166 & 0.109 \\
\hline \multicolumn{7}{|l|}{ Sex } \\
\hline Male & $56(57.1 \%)$ & $131(67.2 \%)$ & $56(65.1 \%)$ & 0.092 & 0.269 & 0.736 \\
\hline \multicolumn{7}{|l|}{ Infection type } \\
\hline Respiratory tract & $23(23.5 \%)$ & $66(33.9 \%)$ & 18 (20.9\%) & 0.068 & 0.68 & 0.029 \\
\hline Bloodstream & 45 (45.9\%) & $35(18.0 \%)$ & $7(8.1 \%)$ & 0.000 & 0.000 & 0.034 \\
\hline Urinary tract & $9(9.2 \%)$ & $46(23.6 \%)$ & 18 (20.9\%) & 0.003 & 0.025 & 0.624 \\
\hline Cerebra & $5(5.1 \%)$ & $3(1.5 \%)$ & $0(0.0 \%)$ & 0.123 & 0.034 & 0.555 \\
\hline Skin and soft tissue & $3(3.1 \%)$ & $16(8.2 \%)$ & $22(25.6 \%)$ & 0.092 & 0.000 & 0.000 \\
\hline Oral cavity & $5(5.1 \%)$ & $3(1.5 \%)$ & $1(1.2 \%)$ & 0.123 & 0.217 & 1.000 \\
\hline Other & $8(8.2 \%)$ & $16(8.2 \%)$ & $11(12.8 \%)$ & 0.990 & 0.303 & 0.229 \\
\hline liver & $0(0.0 \%)$ & $1(0.5 \%)$ & $4(4.7 \%)$ & 1.000 & 0.031 & 0.032 \\
\hline Biliary tract & $0(0.0 \%)$ & $9(4.6 \%)$ & $5(5.8 \%)$ & 0.032 & 0.016 & 0.767 \\
\hline \multicolumn{7}{|l|}{ Underlying disease } \\
\hline Solid tumor & $7(7.1 \%)$ & $20(10.3 \%)$ & $5(5.8 \%)$ & 0.385 & 0.716 & 0.264 \\
\hline Diabetes mellitus & $8(8.2 \%)$ & $34(17.4 \%)$ & $24(27.9 \%)$ & 0.033 & 0.000 & 0.055 \\
\hline Pulmonary disease & $1(1.0 \%)$ & $7(3.6 \%)$ & $5(5.8 \%)$ & 0.276 & 0.099 & 0.522 \\
\hline Hematological malignancy & $25(25.5 \%)$ & $23(11.8 \%)$ & $3(3.5 \%)$ & 0.003 & 0.000 & 0.027 \\
\hline Brain tumor & $10(10.2 \%)$ & $7(3.6 \%)$ & $2(2.3 \%)$ & 0.022 & 0.031 & 0.727 \\
\hline Cerebral vascular disease & $13(13.3 \%)$ & $47(24.1 \%)$ & $10(11.6 \%)$ & 0.030 & 0.738 & 0.017 \\
\hline Hepatobiliary disease & $7(7.1 \%)$ & $13(6.7 \%)$ & $8(9.3 \%)$ & 0.879 & 0.593 & 0.439 \\
\hline Chronic kidney disease & $4(4.1 \%)$ & $21(10.8 \%)$ & $7(8.1 \%)$ & 0.053 & 0.247 & 0.498 \\
\hline Cardiovascular disease & $4(4.1 \%)$ & $20(10.3 \%)$ & $5(5.8 \%)$ & 0.069 & 0.736 & 0.228 \\
\hline Hypertension & $18(18.4 \%)$ & $61(31.3 \%)$ & $17(19.8 \%)$ & 0.019 & 0.852 & 0.047 \\
\hline Immunosuppression & $29(29.6 \%)$ & $38(19.5 \%)$ & $6(7.0 \%)$ & 0.052 & 0.000 & 0.008 \\
\hline \multicolumn{7}{|l|}{ Prior antibiotic exposure } \\
\hline Any antibiotic & $53(54.1 \%)$ & $115(59.0 \%)$ & $0(0.0 \%)$ & 0.424 & 0.000 & 0.000 \\
\hline 1st or 2nd generation cephalosporin & $6(6.1 \%)$ & $7(3.6 \%)$ & $0(0.0 \%)$ & - & - & - \\
\hline 3rd or 4th Generation cephalosporin & $10(10.2 \%)$ & $17(8.7 \%)$ & $0(0.0 \%)$ & - & - & - \\
\hline$\beta$-lactam and $\beta$-lactamase inhibitor & $14(14.3 \%)$ & $19(9.7 \%)$ & $0(0.0 \%)$ & - & - & - \\
\hline Carbapenem & $17(17.4 \%)$ & $21(10.8 \%)$ & $0(0.0 \%)$ & - & - & - \\
\hline Fluoroquinolone & $2(2.0 \%)$ & $5(2.6 \%)$ & $0(0.0 \%)$ & - & - & - \\
\hline Aminoglycoside & $2(2.0 \%)$ & $3(1.5 \%)$ & $0(0.0 \%)$ & - & - & - \\
\hline Tigecycline & $3(3.1 \%)$ & $3(1.5 \%)$ & $0(0.0 \%)$ & - & - & - \\
\hline Glycopeptide & $7(7.1 \%)$ & $12(6.2 \%)$ & $0(0.0 \%)$ & - & - & - \\
\hline Metronidazole & $0(0.0 \%)$ & $1(0.5 \%)$ & $0(0.0 \%)$ & - & - & - \\
\hline 30-day crude mortality & $19(19.4 \%)$ & $24(12.3 \%)$ & $4(4.7 \%)$ & 0.106 & 0.003 & 0.048 \\
\hline
\end{tabular}

Data are presented as number (\%)

Other included renal drainage fluid, amniotic fluid, ascites, and pleural fluid specimen

Bold values suggest statistical significance

- not applicable 
Table 2 Multivariate analysis of variables associated with $K$. pneumoniae infections

\begin{tabular}{|c|c|c|c|c|}
\hline variable & Univariate OR $(95 \% \mathrm{Cl})$ & $P$-value & Mutivariate OR $(95 \% \mathrm{Cl})$ & $P$-value \\
\hline \multicolumn{5}{|l|}{ Nosocomial versus HCA } \\
\hline$\leq 1$ & $6.160(2.668-14.224)$ & 0.000 & $5.665(2.277-14.092)$ & 0.000 \\
\hline $2-10$ & $7.040(2.203-22.497)$ & 0.001 & $5.332(1.506-18.870)$ & 0.009 \\
\hline $11-18$ & 24.640 (2.894-209.815) & 0.003 & $18.772(2.09-168.583)$ & 0.009 \\
\hline $19-59$ & $1.384(0.766-2.502)$ & 0.282 & $1.308(0.699-2.445)$ & 0.401 \\
\hline Diabetes mellitus & $0.037(0.187-0.948)$ & 0.037 & $0.609(0.249-1.487)$ & 0.276 \\
\hline Hematological malignancy & $2.425(1.287-4.570)$ & 0.006 & $1.882(0.686-5.166)$ & 0.220 \\
\hline Brain tumor & $3.052(1.124-8.284)$ & 0.029 & $3.411(1.139-10.215)$ & 0.028 \\
\hline Cerebral vascular disease & $0.525(0.273-1.009)$ & 0.053 & $0.841(0.395-1.792)$ & 0.654 \\
\hline Hypertension & $0.528(0.294-0.949)$ & 0.033 & $1.189(0.592-2.391)$ & 0.626 \\
\hline Immunosuppression & $1.653(0.941-2.904)$ & 0.081 & $0.912(0.37-2.249)$ & 0.912 \\
\hline \multicolumn{5}{|l|}{ Nosocomial versus CA } \\
\hline$\leq 1$ & $5.040(1.660-15.299)$ & 0.004 & $4.456(1.405-14.135)$ & 0.011 \\
\hline $2-10$ & $12.000(1.436-100.278)$ & 0.022 & $4.727(0.494-45.221)$ & 0.178 \\
\hline $11-18$ & $4.800(0.933-24.692)$ & 0.061 & $1.981(0.327-12.001)$ & 0.457 \\
\hline $19-59$ & $0.850(0.427-1.693)$ & 0.644 & $0.671(0.312-1.442)$ & 0.307 \\
\hline Diabetes mellitus & $0.230(0.097-0.544)$ & 0.001 & $0.342(0.129-0.906)$ & 0.031 \\
\hline Hematological malignancy & $9.475(2.747-32.681)$ & 0.000 & $4.467(0.933-21.395)$ & 0.061 \\
\hline Brain tumor & $4.773(1.016-22.427)$ & 0.048 & $7.865(1.554-39.817)$ & 0.013 \\
\hline Immunosuppression & $5.604(2.197-14.291)$ & 0.000 & $2.762(0.807-9.461)$ & 0.106 \\
\hline \multicolumn{5}{|l|}{ HCA versus CA } \\
\hline Diabetes mellitus & $0.546(0.300-0.993)$ & 0.047 & $0.554(0.288-1.067)$ & 0.077 \\
\hline Hematological malignancy & $3.700(1.080-12.674)$ & 0.037 & $2.62(0.664-10.344)$ & 0.169 \\
\hline Cerebral vascular disease & $2.414(1.156-5.041)$ & 0.019 & $2.486(1.154-5.356)$ & 0.02 \\
\hline Hypertension & $1.848(1.003-3.404)$ & 0.049 & $2.08(1.065-4.061)$ & 0.032 \\
\hline Immunosuppression & $3.227(1.309-7.954)$ & 0.011 & $2.661(0.971-7.297)$ & 0.057 \\
\hline
\end{tabular}

Bold values suggest statistical significance

infections. In multivariate analysis, hematological malignancy $(\mathrm{OR}=4.467)$ and brain tumor $(\mathrm{OR}=7.865)$ were independent variables associated with nosocomial cases compared to CA cases, whereas diabetes mellitus was more frequently associated with $C A$ cases $(\mathrm{OR}=0.342)$. Cerebral vascular disease $(\mathrm{OR}=2.486)$ and hypertension $(\mathrm{OR}=2.080)$ were independent variables associated with HCA infections compared to CA infections.

Both patients with nosocomial and HCA infections presented a higher prevalence of prior antibiotic exposure, accounting for $54.1 \%$ and $59.0 \%$, respectively. No prior antibiotic exposure was observed in CA infection patients. Patients with nosocomial (19.4\%) and HCA (12.3\%) infections exhibited higher rates of 30-day mortality than patients with CA infections (4.7\%).

\section{Antimicrobial resistance of nosocomial, HCA, and CA $K$. pneumoniae isolates}

The distribution of the percentage of antimicrobialresistant K. pneumoniae strains isolated from the three groups is summarized in Table 3. Overall, the resistance rates to most of the antibiotics tested were similar for nosocomial isolates and HCA isolates $(P>0.05)$, and both the strains were significantly more resistant than the CA strains $(P<0.05)$. The percentages of CRKP strains, ESBL-producing strains and MDR strains were similar between the nosocomial and HCA cases and were higher in both cases than in CA cases: (nosocomial vs. HCA vs. CA) for CRKP strains ( $22.5 \%$ vs. $28.7 \%$ vs. $4.7 \%)$, ESBLproducing strains ( $40.8 \%$ vs. $35.9 \%$ vs. $24.4 \%)$, and MDR strains ( $53.1 \%$ vs. $52.3 \%$ vs. $23.3 \%)$.

\section{Distribution of carbapenem-resistance genes and ESBL genes}

A total of 82 CRKP isolates were identified from the three groups. The distribution of carbapenem resistance genes of the CRKP isolates among the three groups is shown in Table 4 . Only 3 carbapenem resistance genes, $b l a_{\mathrm{KPC}}$ $(80.5 \%, 66 / 82), b l a_{\mathrm{NDM}}(14.6 \%, 12 / 82)$, and $b l a_{\mathrm{OXA}-48-\text { like }}$ $(3.7 \%, 3 / 82)$, were identified among the 82 CRKP strains. Four strains presented simultaneously $b l a_{\mathrm{KPC}}$ and $b l a_{\mathrm{NDM}}$ 
Table 3 Antimicrobial resistance rates of $K$. pneumoniae strains

\begin{tabular}{|c|c|c|c|c|c|c|}
\hline \multirow[t]{2}{*}{ Antimicrobials } & \multirow{2}{*}{$\begin{array}{l}\text { Nosocomial } \\
(n=98)\end{array}$} & \multirow{2}{*}{$\begin{array}{l}\text { HCA } \\
(n=195)\end{array}$} & \multirow{2}{*}{$\begin{array}{l}\text { CA } \\
(n=86)\end{array}$} & \multicolumn{3}{|l|}{$P$-value } \\
\hline & & & & $\begin{array}{l}\text { HCA } \\
\text { versus Nosocomial }\end{array}$ & $\begin{array}{l}\text { CA } \\
\text { versus Nosocomial }\end{array}$ & CA versus $\mathrm{HCA}$ \\
\hline Ampicillin & $96(98.0 \%)$ & 195 (100.0\%) & $82(95.4 \%)$ & 0.111 & 0.420 & 0.002 \\
\hline Cefazolin & $68(69.4 \%)$ & $131(67.2 \%)$ & $36(41.9 \%)$ & 0.702 & 0.000 & 0.000 \\
\hline Cefuroxime & $58(59.2 \%)$ & $114(58.5 \%)$ & $27(31.4 \%)$ & 0.906 & 0.000 & 0.000 \\
\hline Ceftriaxone & $56(57.1 \%)$ & 109 (55.9\%) & $26(30.2 \%)$ & 0.839 & 0.000 & 0.000 \\
\hline Ceftazidime & $43(43.9 \%)$ & $85(43.6 \%)$ & $18(20.9 \%)$ & 0.963 & 0.001 & 0.000 \\
\hline Cefepime & $41(41.8 \%)$ & $87(44.6 \%)$ & $15(17.4 \%)$ & 0.651 & 0.000 & 0.000 \\
\hline Imipenem & $20(20.4 \%)$ & $57(29.2 \%)$ & $4(4.7 \%)$ & 0.105 & 0.002 & 0.000 \\
\hline Meropenem & $21(21.4 \%)$ & $57(29.2 \%)$ & $4(4.7 \%)$ & 0.154 & 0.001 & 0.000 \\
\hline Ertapenem & $24(24.5 \%)$ & $58(29.7 \%)$ & $4(4.7 \%)$ & 0.345 & 0.000 & 0.000 \\
\hline Amoxicillin/Clavulanic Acid & $44(44.9 \%)$ & $78(40.0 \%)$ & $13(15.1 \%)$ & 0.422 & 0.000 & 0.000 \\
\hline Cefoperazone/Sulbactam & $41(41.8 \%)$ & $66(33.9 \%)$ & $9(10.5 \%)$ & 0.180 & 0.000 & 0.000 \\
\hline Piperacillin/Tazobactam & $43(43.9 \%)$ & $74(38.0 \%)$ & $8(9.3 \%)$ & 0.328 & 0.000 & 0.000 \\
\hline Aztreonam & $47(48.0 \%)$ & $102(52.3 \%)$ & $22(25.6 \%)$ & 0.482 & 0.002 & 0.000 \\
\hline Cefoxitin & $31(31.6 \%)$ & $73(37.4 \%)$ & $11(12.8 \%)$ & 0.327 & 0.002 & 0.000 \\
\hline Ciprofloxacin & 45 (45.9\%) & $103(52.8 \%)$ & $21(24.4 \%)$ & 0.265 & 0.002 & 0.000 \\
\hline Levofloxacin & $31(31.6 \%)$ & $90(46.2 \%)$ & $14(16.3 \%)$ & 0.017 & 0.016 & 0.000 \\
\hline Amikacin & $36(36.7 \%)$ & $46(23.6 \%)$ & $6(7.0 \%)$ & 0.018 & 0.000 & 0.001 \\
\hline Gentamicin & $47(48.0 \%)$ & $91(46.6 \%)$ & $24(27.9 \%)$ & 0.834 & 0.005 & 0.004 \\
\hline Trimethoprim/Sulfamethoxazole & $52(53.1 \%)$ & $106(54.4 \%)$ & $34(39.5 \%)$ & 0.833 & 0.067 & 0.028 \\
\hline Tigecycline & $1(1.0 \%)$ & $4(2.1 \%)$ & $1(1.2 \%)$ & 0.667 & 1.000 & 1.000 \\
\hline ESBL-production & $40(40.8 \%)$ & $70(35.9 \%)$ & $21(24.4 \%)$ & 0.412 & 0.018 & 0.072 \\
\hline Multidrug resistance & $52(53.1 \%)$ & $102(52.3 \%)$ & $20(23.3 \%)$ & 0.903 & 0.000 & 0.000 \\
\hline
\end{tabular}

Data are presented as number (\%)

Bold values suggest statistical significance

Table 4 Carbapenemase resistance genes distribution among CRKP strains

\begin{tabular}{|c|c|c|c|c|c|c|c|}
\hline \multirow[t]{2}{*}{ Carbapenemase genes } & \multirow{2}{*}{$\begin{array}{l}\text { Total } \\
(n=82)\end{array}$} & \multirow{2}{*}{$\begin{array}{l}\text { Nosocomial } \\
(n=22)\end{array}$} & \multirow{2}{*}{$\begin{array}{l}\text { HCA } \\
(n=56)\end{array}$} & \multirow{2}{*}{$\begin{array}{l}C A \\
(n=4)\end{array}$} & \multicolumn{3}{|l|}{$P$-value } \\
\hline & & & & & $\begin{array}{l}\text { HCA } \\
\text { versus Nosocomial }\end{array}$ & $\begin{array}{l}\text { CA } \\
\text { versus Nosocomial }\end{array}$ & CA versus $H C A$ \\
\hline$b / a_{\mathrm{KPC}}$ & $66(80.5 \%)$ & $19(86.4 \%)$ & $44(78.6 \%)$ & $3(75.0 \%)$ & 0.563 & 0.511 & 1.000 \\
\hline$b / a_{\mathrm{NDM}}$ & $12(14.6 \%)$ & $2(9.1 \%)$ & $9(16.1 \%)$ & $1(25.0 \%)$ & 0.719 & 0.408 & 0.528 \\
\hline$b / a_{\text {OXA-48-like }}$ & $3(3.7 \%)$ & $1(4.6 \%)$ & $2(3.6 \%)$ & $0(0.0 \%)$ & - & - & - \\
\hline$b / a_{\mathrm{KPC}}$ and $b / a_{\mathrm{NDM}}$ & $4(4.9 \%)$ & $1(4.6 \%)$ & $3(5.4 \%)$ & $0(0.0 \%)$ & - & - & - \\
\hline None detected & $5(6.1 \%)$ & $1(4.6 \%)$ & $4(7.1 \%)$ & $0(0.0 \%)$ & - & - & - \\
\hline
\end{tabular}

Data are presented as number (\%)

- : not applicable

genes. Five isolates were negative for the screened carbapenemase genes.

PCR showed that all nosocomial, HCA, and CA ESBL-positive strains harbored at least one of the ESBL gene groups, with positive rates of $91.6 \%(120 / 131)$ for bla $_{\mathrm{SHV}}, 64.9 \%(85 / 131)$ for bla $_{\mathrm{TEM}}, 51.9 \%(68 / 131)$ for $b l a_{\text {CTX-M-9-group }}, 42.0 \%$ (55/131) for $b l a_{\text {CTX-M-1-group }}$ (Table 5). The $b l a_{\mathrm{CTX}-\mathrm{M}-2 \text {-group }}$ gene was not detected in
ESBL-producing strains. No significant differences were found in the distribution of $b l a_{\mathrm{SHV}}, b l a_{\mathrm{TEM}}$, and $b l a_{\mathrm{OXA}-1-}$ like genes among the nosocomial, HCA, and CA infection groups. However, the $b l a_{\mathrm{CTX}-\mathrm{M} \text {-1-group }}$ and $b l a_{\mathrm{CTX} \text {-M-9-group }}$ genes were disproportionately distributed between nosocomial, HCA, and CA isolates (Table 5). Fifty-one of the 131 ESBL-positive strains were observed to co-harbor $b l a_{\mathrm{SHV}}$ and $b l a_{\mathrm{CTX}-\mathrm{M}-1 \text {-group}}$, with $74.5 \%$ (38/51) having 
Table 5 Distribution of ESBLs genes among three groups of ESBL-producing strains

\begin{tabular}{|c|c|c|c|c|c|c|c|}
\hline \multirow[t]{3}{*}{ ESBLs genes } & \multirow{3}{*}{$\begin{array}{l}\text { Total } \\
(n=131)\end{array}$} & \multirow{3}{*}{$\begin{array}{l}\text { Nosocomial } \\
(n=40)\end{array}$} & \multirow{3}{*}{$\begin{array}{l}\text { HCA } \\
(n=70)\end{array}$} & \multirow{3}{*}{$\begin{array}{l}C A \\
(n=21)\end{array}$} & \multicolumn{3}{|l|}{$P$-value } \\
\hline & & & & & HCA versus & CA versus & $C A$ versus \\
\hline & & & & & Nosocomial & Nosocomial & HCA \\
\hline$b l a_{S H V}$ & $120(91.6 \%)$ & $34(85.0 \%)$ & $67(95.7 \%)$ & $19(90.5 \%)$ & 0.070 & 0.703 & 0.326 \\
\hline$b / a_{\mathrm{TEM}}$ & $85(64.9 \%)$ & $25(62.5 \%)$ & $45(64.3 \%)$ & $15(71.4 \%)$ & 0.851 & 0.486 & 0.545 \\
\hline$b l a_{\mathrm{OXA}-1-\text { like }}$ & $6(4.6 \%)$ & $0(0.0 \%)$ & $5(7.1 \%)$ & $1(4.8 \%)$ & 0.157 & 0.344 & 1.000 \\
\hline bla $a_{\text {CTX-M-group }}$ & $123(93.9 \%)$ & $39(97.5 \%)$ & $65(92.9 \%)$ & $19(90.5 \%)$ & 0.414 & 0.27 & 0.66 \\
\hline bla $a_{\mathrm{CTX}-\mathrm{M}-1 \text {-group }}$ & $55(42.0 \%)$ & $10(25.0 \%)$ & $31(44.3 \%)$ & $14(66.7 \%)$ & 0.044 & 0.002 & 0.072 \\
\hline 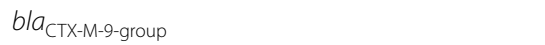 & $68(51.9 \%)$ & $26(65.0 \%)$ & $36(51.4 \%)$ & $6(28.6 \%)$ & 0.167 & 0.007 & 0.065 \\
\hline$b / a_{\mathrm{SHV}}$ and $b / a_{\mathrm{CTX}-\mathrm{M}-1-\text { group }}$ & $51(38.9 \%)$ & $8(20.0 \%)$ & $30(42.9 \%)$ & $13(61.9 \%)$ & 0.015 & 0.001 & 0.125 \\
\hline$b / a_{\mathrm{SHV}}$ and $b / a_{\mathrm{CTX}-\mathrm{M}-9 \text {-group }}$ & 61 (46.6\%) & $22(55.0 \%)$ & $34(48.6 \%)$ & $5(23.8 \%)$ & 0.516 & 0.020 & 0.044 \\
\hline$b / a_{\mathrm{TEM}}$ and $b / a_{\mathrm{CTX}-\mathrm{M} \text {-1-group }}$ & $42(32.1 \%)$ & $9(22.5 \%)$ & $23(32.9 \%)$ & $10(47.6 \%)$ & 0.250 & 0.440 & 0.217 \\
\hline$b / a_{\mathrm{TEM}}$ and bla $a_{\mathrm{CTX}-\mathrm{M} \text {-9-group }}$ & $40(30.5 \%)$ & $15(37.5 \%)$ & $21(30.0 \%)$ & $4(19.1 \%)$ & 0.420 & 0.139 & 0.324 \\
\hline$b l a_{C T X-M-9-g r o u p}$ and bla $a_{C T X-M-1-\text { group }}$ & $8(6.1 \%)$ & $2(5.0 \%)$ & $5(7.1 \%)$ & $1(4.8 \%)$ & - & - & - \\
\hline bla $a_{\mathrm{SHV}}, b / a_{\mathrm{TEM}}$ and bla $a_{\mathrm{CTX}-\mathrm{M}-9-\text { group }}$ & $36(27.5 \%)$ & $13(32.5 \%)$ & $20(28.6 \%)$ & $3(14.3 \%)$ & 0.665 & 0.124 & 0.186 \\
\hline bla $a_{\mathrm{SHV}}, b / a_{\mathrm{TEM}}$ and bla $a_{\mathrm{CTX}-\mathrm{M}-1 \text {-group }}$ & $38(29.0 \%)$ & $7(17.5 \%)$ & $22(31.4 \%)$ & $9(42.9 \%)$ & 0.111 & 0.032 & 0.332 \\
\hline bla $a_{\mathrm{SHV}}, b / a_{\mathrm{CTX} \text {-M-9-group }}$ and bla $a_{\mathrm{CTX}-\mathrm{M}-1 \text {-group }}$ & $8(6.1 \%)$ & $2(5.0 \%)$ & $5(7.1 \%)$ & $1(4.8 \%)$ & - & - & - \\
\hline bla $a_{\mathrm{TEM}}, b / a_{\mathrm{CTX}-\mathrm{M}-9 \text {-group }}$ and $b / a_{\mathrm{CTX}-\mathrm{M}-1 \text {-group }}$ & $6(4.6 \%)$ & $2(5.0 \%)$ & $3(4.3 \%)$ & $1(4.8 \%)$ & - & - & - \\
\hline bla $a_{\mathrm{SHV}}, b / a_{\mathrm{TEM}}, b / a_{\mathrm{CTX}-\mathrm{M}-9 \text {-group }}$ and bla $a_{\mathrm{CTX}-\mathrm{M}-1 \text {-group }}$ & $6(4.6 \%)$ & $2(5.0 \%)$ & $3(4.3 \%)$ & 1 (4.8\%) & - & - & - \\
\hline
\end{tabular}

Data are presented as number (\%)

Bold values suggest statistical significance

not applicable

co-existence of $b l a_{\mathrm{SHV}}, b l a_{\mathrm{TEM}}$ and $b l a_{\mathrm{CTX}-\mathrm{M}-1 \text {-group, }}$ and these strains were more commonly identified in the CA group than in the nosocomial group $(61.9 \%$ vs. $20.0 \%$, $P=0.001 ; 42.9 \%$ vs. $17.5 \%, P=0.032$, respectively). Conversely, strains possessing both $b l a_{\mathrm{SHV}}$ and $b l a_{\mathrm{CTX}-\mathrm{M}}$ 9-group were more likely to be found in the nosocomial infection group than in the CA group $(55.0 \%$ vs. $23.8 \%$, $P=0.02)$

\section{Pulsed-field gel electrophoresis}

All CRKP or ESBL-producing strains were subjected to PFGE for assessing the clonality of the strains. Three ESBL-producing isolates failed to be genotyped by PFGE. Fifty-six nosocomial strains were divided into 36 clusters at a cut-off of $80 \%$ similarity, with the major cluster $\mathrm{C} 13$ accounting for 14 ESBL-producing strains (Fig. 1). One hundred and three HCA strains were separated into 65 clusters, with two major clusters C82 and C83, accounting for 17 and 10 CRKP strains respectively. Twenty-four CA strains distributed among 22 clusters. Despite the high genetic diversity, several sets of identical profiles were found among the three groups. In PFGE cluster C13, three sets of identical profiles were observed, and 9 of which were nosocomial strains. In PFGE cluster C82, two sets of identical PFGE profiles were detected among HCA isolates. In cluster C82-II, four HCA isolates and one nosocomial strain showed $100 \%$ similarity. Two HCA isolates and one nosocomial strain in cluster C82-III also shared complete similarity. In addition, we observed that some strains with the same PFGE profiles but carried different resistance gene profiles. For instance, isolate $268,182,255$, and 166 in cluster C13 presented identical profiles, but carried different bla genes. Isolate 98 carrying $b l a_{\text {KPC }}$ showed $100 \%$ similarity to isolate 49 carrying $b l a_{\mathrm{NDM}}$.

\section{Discussion}

In the current study, we used the definition proposed by Friedman et al. [2] to define healthcare-associated $K$. pneumoniae infections, and we found that HCA infections accounted for over half of the K. pneumoniae infections. HCA infections showed similar clinical features with nosocomial infections. CA infections presented different characteristic from both HCA and nosocomial infections. Hematological malignancy was more commonly related to nosocomial infections, whereas diabetes mellitus was more frequently associated with $\mathrm{CA}$ infections. These findings were also observed in previous reports from Taiwan and Korea [23, 24]. It has been reported that cancer was more commonly associated with HCA $K$. pneumoniae bacteremia patients than CA bacteremia patients $[4,5,25]$. In the present study, 


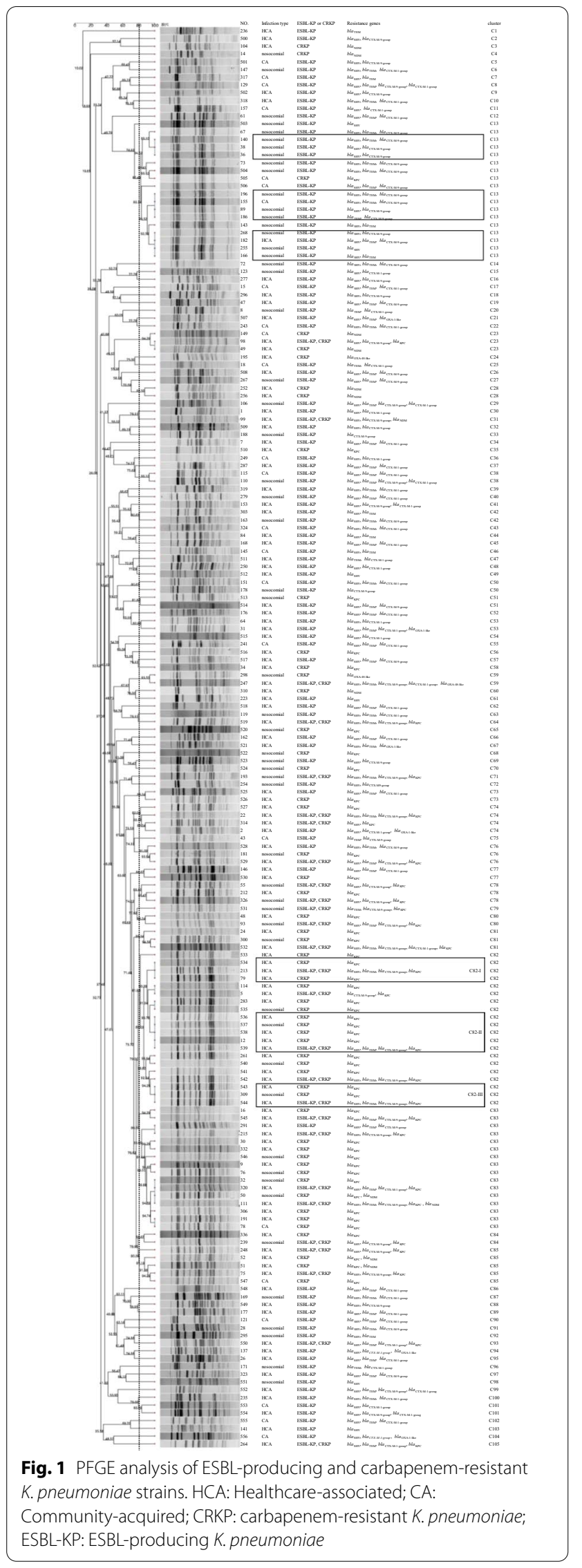

however, cerebral vascular disease and hypertension were more associated with HCA infections compared to CA infections. This discrepancy may be due to the regional differences in underlying diseases. Indeed, cerebral vascular disease and hypertension are epidemics in Chinese communities [26, 27]. Patients with these diseases have a greater demand for medical care services.

In this study, the 30-day mortality rates were similar for patients with nosocomial and HCA infections and both patients exhibited significantly higher 30-day mortality rates than CA infection patients. These results were similar to other studies $[4,5]$ and may largely be due to the variation in underlying diseases between the three groups, which are classical risk factors for prognosis of infectious diseases.

An important finding in this study was that HCA K. pneumoniae strains exhibited similar antimicrobial resistance patterns with nosocomial strains, and both the nosocomial and HCA strains were significantly more resistant than the CA strains. This observation indicates that clinicians should consider similar empirical therapy for patients with nosocomial and HCA K. pneumoniae infections. A recent study in Taiwan reported that the rate of resistance in the group of HCA $K$. pneumoniae bacteremia was between those groups of nosocomial and CA bacteremia [28]. This discrepancy may be due to the different infection types of the population studied, or the regional differences of resistance characteristics in K. pneumoniae. In our study, $29.7 \%$ of the HCA isolates were carbapenem resistant, $35.9 \%$ expressed ESBL, and $52.3 \%$ were MDR, indicating the severe drug resistance in isolates from the HCA infections. The high degree of antibiotic resistance in HCA strains may be related to the frequent contact with healthcare facilities among HCA patients, where the drug-resistant bacteria could spread. In addition, other factors such as overuse of antibiotics might also responsible for developing antimicrobial resistance. Our results suggest that increased attention should be paid to the HCA K. pneumoniae infections.

The spread of CRKP strains has become a new public health crisis worldwide. Published studies on the distribution of CRKP isolates among nosocomial, HCA, and $\mathrm{CA}$ infections are scarce. The present study showed that CRKP strains were rare in the CA group, but more prevalent in both HCA and nosocomial groups. Thus, it should be of caution when use carbapenems to treat patients with HCA and nosocomial infections. In agreement with other studies, the $b l a_{\mathrm{KPC}}$ was the dominant carbapenemase gene carried by CRKP strains [18, 29]. In this investigation, only 12 and 3 CRKP strains harbored $b l a_{\mathrm{NDM}}$ and $b l a_{\mathrm{OXA}-48-\text { like }}$ genes respectively. Indeed, the $b l a_{\text {OXA-48-like }}$ producers were more prevalent in western countries such as France, Spain and Germany, but were 
rare in China [30-32]. In addition, the bla genes screened were absent in five CRKP isolates. Among these strains, only one showed carbapemase activity in the modified carbapenem inactivation method test (data not shown), indicating other resistance genes not investigated may mediate the carbapemase activity. The remaining four CRKP may have other resistance mechanisms contributing to carbapenem resistance. The production of ESBLs combined with mutations in or loss of the porin Ompk35 or Ompk36 can be associated with carbapenem resistance $[33,34]$. In addition, the overexpression of efflux pump in strains may also lead to carbapenem resistance. However, both porin Ompk35 and Ompk36 were present in the other four CRKP strains with ESBL-positive in this study (data not shown). Further studies should be conducted to assess the presence of the mutations in strains isolated in the current investigation.

This study showed that the ESBL-producing strains were prevalent both in medical and community settings. Previous studies by Zhang et al. [35] and Quan et al. [36] also reported a high prevalence of ESBL-positive strains in community-onset $K$. pneumoniae infections. These data suggest that ESBL producers have disseminated to the community, which poses a challenge to resistance control. $B l a_{\text {СTX-M }}$ was the most frequently identified ESBL gene among ESBL producers in our study. This is accordance with the fact that CTX-M is the most common ESBL genotype in China [36]. No significant differences were observed in the distribution of $b l a_{\text {СТX-M, }}$, $b l a_{\mathrm{SHV}}$ and $b l a_{\mathrm{TEM}}$ genes among isolates from the three groups, suggesting the wide dissemination of these genes possibly via horizontal transfer in different populations. Interestingly, the CTX-M-1 variant was more frequently detected among CA strains than among nosocomial strains, whereas the CTX-M-9 variant was more common in nosocomial strains than in CA strains. This suggests that nosocomial and CA ESBL-producing strains may carry different plasmids, which carried the different subtype of CTX-M genes.

Overall, the PFGE typing showed a high clonal diversity among the ESBL-producing and CRKP strains isolated from the three different groups, indicating the variety of infection sources acquired by the three populations. Of note, the clonal dissemination of ESBLproducing strains has occurred in the hospital as indicated by the three identical profiles observed in cluster C13. Further study is needed to investigate the origin and the route of the transmission. In cluster C82-II and cluster C82-III, the HCA strains and nosocomial strains showed the same PFGE profiles, suggesting the cross transmission of CRKP among HCA and nosocomial patients. CRKP strains could be introduced into the hospital, then spread within the institution and might cause nosocomial infections during the entry of $\mathrm{HCA}$ patients with CRKP infections, of which we should keep alert. Identical profiles among HCA CRKP isolates implies the clonal spread of $K$. pneumoniae among HCA patients. It suggests the healthcare-associated transmission of CRKP strains in the local area. In addition, the same PFGE profiles were observed in strains carrying different resistance gene profiles, suggesting later acquisition of the resistance genes via horizontal transfer under antibiotic selection pressure.

The main finding in the present study was that the antimicrobial resistance of HCA isolates was similar in severity to nosocomial isolates. This study was conducted at a tertiary hospital in Guangzhou city, Guangdong province, where more severe patients from all over the province or neighboring provinces had been hospitalized and approximately $20 \%$ had been referred from other hospitals, which could explain the serious drug resistance in the HCA group in our study. Given distinct features of $K$. pneumoniae strains from different regions, more epidemiological data on nosocomial, HCA, and CA K. pneumoniae infections are needed to better manage patients.

\section{Conclusions}

HCA K. pneumoniae infections were similar to nosocomial infections regarding clinical features and antimicrobial resistance, and were different to $C A$ infections. CRKP and ESBL-producing strains were mainly prevalent in patients with HCA and nosocomial infections, showing genetic diversity. Identical profiles observed in HCA and nosocomial CRKP strains suggested the transmission of CRKP among HCA and nosocomial patients. These findings indicate that empirical antimicrobial treatment for patients with HCA K. pneumoniae infections should be similar to those for patients with nosocomial infections and great attention should be paid to these infections due to the possible dissemination of antimicrobial resistance among them.

\section{Supplementary Information}

The online version contains supplementary material available at https://doi. org/10.1186/s13756-021-00910-1.

Additional file 1: Table S1. Primer sequences of resistant genes for $K$. pneumoniae.

Abbreviations

PCR: Polymerase chain reaction; CRKP: Carbapenem-resistant K. pneumoniae; ESBL: Extended-spectrum beta-lactamase; MDR: Multidrug resistant; PFGE: Pulsed-field gel electrophoresis; HCA: Healthcare-associated; CA: Community-acquired.

Acknowledgements

Not applicable. 


\section{Authors' contributions}

$\mathrm{JH}$ conceived and designed the study. T-TL collected the isolates and performed the experiments. C-YZ participated in the data collection. T-TL analyzed the data and wrote the manuscript. JH, Ling W, and L-WF revised the manuscript. All authors read and approved the final manuscript.

\section{Funding}

This work was funded by Guangdong Basic and Applied Basic Research Foundation (2020A1515010333).

\section{Availability of data and materials}

All materials and data analyzed during this study are contained within the manuscript.

\section{Ethics approval and consent to participate}

The study protocol was approved by the Institutional Review Board of Zhujiang Hospital. Informed consent was not obtained due to the retrospective nature of the study.

\section{Consent for publication}

Not applicable.

\section{Competing interests}

The authors declare that they have no competing interests.

\section{Author details}

${ }^{1}$ Department of Nosocomial Infection Administration, Zhujiang Hospital, Southern Medical University, Guangzhou 510282, Guangdong, China. ${ }^{2}$ Department of Laboratory Medicine, Zhujiang Hospital, Southern Medical University, Guangzhou 510282, Guangdong, China. ${ }^{3}$ School of Public Health (Shenzhen), Sun Yat-Sen University, Shenzhen 518000, Guangdong, China. ${ }^{4}$ Department of Infectious Disease, Nanfang Hospital, Guangzhou 510282, Guangdong, China.

Received: 8 December 2020 Accepted: 15 February 2021

Published online: 25 February 2021

\section{References}

1. Paczosa MK, Mecsas J. Klebsiella pneumoniae: going on the offense with a strong defense. Microbiol Mol Biol Rev. 2016;80:629-61.

2. Friedman ND, Kaye KS, Stout JE, MCGarry SA, Trivette SL, Briggs JP, et al. Health care-associated bloodstream infections in adults: a reason to change the accepted definition of community-acquired infections. Ann Intern Med. 2002;137:791-7.

3. Aguilar-Duran S, Horcajada JP, Sorlí L, Montero M, Salvadó M, Grau S, et al. Community-onset healthcare-related urinary tract infections: comparison with community and hospital-acquired urinary tract infections. J Infect. 2012:64:478-83.

4. Wu HS, Wang FD, Tseng CP, Wu TH, Lin YT, Fung CP. Characteristics of healthcare-associated and community-acquired Klebsiella pneumoniae bacteremia in Taiwan. J Infect. 2012;64:162-8.

5. Jung Y, Lee MJ, Sin HY, Kim NH, Hwang JH, Park J, et al. Differences in characteristics between healthcare-associated and community-acquired infection in community-onset Klebsiella pneumoniae bloodstream infection in Korea. BMC Infect Dis. 2012;12:239.

6. Wang X, Wang Q, Cao B, Sun S, Zhang Y, Gu B, et al. Retrospective observational study from a Chinese network of the impact of combination therapy versus monotherapy on mortality from carbapenemresistant Enterobacteriaceaebacteremia. Antimicrob Agents Chemother. 2019;63:1673.

7. Nordmann P, Naas T, Poirel L. Global spread of Carbapenemase-producing Enterobacteriaceae. Emerg Infect Dis. 2011;17:1791-8.

8. He SZ, Li Z, Yang Q, Quan ML, Zhao L, Hong Z. Resistance trends among 1294 nosocomial Acinetobacter baumannii strains from a tertiary General Hospital in China, 2014-2017. Clin Lab. 2020;66:325-9.

9. David S, Reuter S, Harris SR, Glasner C, Feltwell T, Argimon S, et al. Epidemic of carbapenem-resistant Klebsiella pneumoniae in Europe is driven by nosocomial spread. Nat Microbiol. 2019;4:1919-29.
10. Yamakawa H, Kosai K, Akamatsu N, Matsuda J, Kaku N, Uno N, et al. Molecular and epidemiological analysis of IMP-1 metallo- $\beta$-lactamaseproducing Klebsiella pneumoniae in a tertiary care hospital in Japan. J Infect Chemother. 2019;25:240-6.

11. Lee CR, Lee JH, Park KS, Kim YB, Jeong BC, Lee SH. Global dissemination of carbapenemase-producing Klebsiella pneumoniae: epidemiology, genetic context, treatment options, and detection methods. Front Microbiol. 2016;7:895

12. Pitout JD, Nordmann P, Poirel L. Carbapenemase-producing Klebsiella pneumoniae, a key pathogen set for global nosocomial dominance. Antimicrob Agents Chemother. 2015;59:5873-84.

13. Rocha FR, Fehlberg LCC, Cordeiro-Moura JR, Ramos AC, Pinto VPT, Barbosa FCB. High frequency of extended-spectrum beta-lactamase-producing Klebsiella pneumoniae nosocomial strains isolated from a teaching Hospital in Brazil. Microb Drug Resist. 2019;25:909-14.

14. Bradford PA. Extended-spectrum beta-lactamases in the 21st century: characterization, epidemiology, and detection of this important resistance threat. Clin Microbiol Rev. 2001;14:933-51.

15. Wu W, Feng Y, Tang G, Qiao F, McNally A, Zong Z. NDM metallo- $\beta$ lactamases and their bacterial producers in health care settings. Clin Microbiol Rev. 2019;32:e00115.

16. Wyres KL, Holt KE. Klebsiella pneumoniae as a key trafficker of drug resistance genes from environmental to clinically important bacteria. Curr Opin Microbiol. 2018:45:131-9.

17. Ferreira RL, da Silva BCM, Rezende GS, Nakamura-Silva R, Pitondo-Silva A, Campanini EB, et al. High prevalence of multidrug-resistant Klebsiella pneumoniae harboring several virulence and $\beta$-lactamase encoding genes in a Brazilian intensive care unit. Front Microbiol. 2018;9:3198.

18. Zeng L, Deng Q, Zeng T, Liu Y, Zhang J, Cao X. Prevalence of Carbapenemresistant Klebsiella pneumoniae infection in Southern China: clinical characteristics, antimicrobial resistance, virulence, and geographic distribution. Microb Drug Resist. 2020;26:483-91.

19. Dallenne C, Da Costa A, Decré D, Favier C, Arlet G. Development of a set of multiplex PCR assays for the detection of genes encoding important beta-lactamases in Enterobacteriaceae. J Antimicrob Chemother. 2010;65:490-5

20. Kiaei S, Moradi M, Hosseini-Nave H, Ziasistani M, Kalantar-Neyestanaki D. Endemic dissemination of different sequence types of carbapenemresistant Klebsiella pneumoniae strains harboring bla $a_{\text {NDM }}$ and 165 rRNA methylase genes in Kerman hospitals, Iran, from 2015 to 2017. Infect Drug Resist. 2019;12:45-54.

21. Wang L, Fu L, Liu Z, Guo H, Wang L, Feng M, et al. Comparative analysis of antimicrobial resistance, integrons, and virulence genes among extended-spectrum $\beta$-lactamase-positive Laribacter hongkongensis from edible frogs and freshwater fish. Microb Drug Resist. 2019;25:855-64.

22. Hong SS, Kim K, Huh JY, Jung B, Kang MS, Hong SG. Multiplex PCR for rapid detection of genes encoding class $A$ carbapenemases. Ann Lab Med. 2012;32:359-61.

23. Tsay RW, Siu LK, Fung CP, Chang FY. Characteristics of bacteremia between community-acquired and nosocomial Klebsiella pneumoniae infection: risk factor for mortality and the impact of capsular serotypes as a herald for community-acquired infection. Arch Intern Med. 2002;162:1021-7.

24. Kang Cl, Kim SH, Bang JW, Kim HB, Kim NJ, Kim EC, et al. Communityacquired versus nosocomial Klebsiella pneumoniae bacteremia: clinical features, treatment outcomes, and clinical implication of antimicrobial resistance. J Korean Med Sci. 2006;21:816-22.

25. Takeshita N, Kawamura I, Kurai H, Araoka H, Yoneyama A, Fujita T, et al Unique characteristics of community-onset healthcare- associated bloodstream infections: a multi-centre prospective surveillance study of bloodstream infections in Japan. J Hosp Infect. 2017;96:29-34.

26. Wang Z, Chen Z, Zhang L, Wang X, Hao G, Zhang Z, et al. Status of hypertension in China: results from the China hypertension survey, 2012-2015. Circulation. 2018:137:2344-56.

27. Wu S, Wu B, Liu M, Chen Z, Wang W, Anderson CS, et al. Stroke in China: advances and challenges in epidemiology, prevention, and management. Lancet Neurol. 2019;18:394-405.

28. Juan $\mathrm{CH}$, Chuang C, Chen CH, Li L, Lin YT. Clinical characteristics, antimicrobial resistance and capsular types of community-acquired, healthcare-associated, and nosocomial Klebsiella pneumoniae bacteremia. Antimicrob Resist Infect Control. 2019:8:1. 
29. Wang Q, Wang Z, Zhang F, Zhao C, Yang B, Sun Z, et al. Long-term continuous antimicrobial resistance surveillance among nosocomial gram-negative Bacilli in China from 2010 to 2018 (CMSS). Infect Drug Resist. 2020;13:2617-29.

30. Messaoudi A, Haenni M, Bouallègue $O$, Saras E, Chatre P, Chaouch C, et al. Dynamics and molecular features of OXA-48-like-producing Klebsiella pneumoniae lineages in a Tunisian hospital. J Glob Antimicrob Resist. 2020;20:87-93.

31. Machuca J, López-Cerero L, Fernández-Cuenca F, Mora-Navas L, Mediavilla-Gradolph C, López-Rodríguez I, et al. OXA-48-like-producing Klebsiella pneumoniae in Southern Spain in 2014-2015. Antimicrob Agents Chemother. 2019;63:1.

32. Wang $Q$, Wang $X$, Wang J, Ouyang $P$, Jin C, Wang R, et al. Phenotypic and genotypic characterization of Carbapenem-resistant Enterobacteriaceae: data from a longitudinal large-scale CRE study in China (2012-2016). Clin Infect Dis. 2018;67:S196-s205.

33. Du J, Cao J, Shen L, Bi W, Zhang X, Liu H, et al. Molecular epidemiology of extensively drug-resistant Klebsiella pneumoniae outbreak in Wenzhou, Southern China. J Med Microbiol. 2016;65:1111-8.
34. Skurnik D, Lasocki S, Bremont S, Muller-Serieys C, Kitzis MD, Courvalin P, et al. Development of ertapenem resistance in a patient with mediastinitis caused by Klebsiella pneumoniae producing an extended-spectrum beta-lactamase. J Med Microbiol. 2010;59:115-9.

35. Zhang J, Zhou K, Zheng B, Zhao L, Shen P, Ji J, et al. High prevalence of ESBL-producing Klebsiella pneumoniae causing community-onset infections in China. Front Microbiol. 2016;7:1830.

36. Quan J, Zhao D, Liu L, Chen Y, Zhou J, Jiang Y, et al. High prevalence of ESBL-producing Escherichia coli and Klebsiella pneumoniae in community-onset bloodstream infections in China. J Antimicrob Chemother. 2017;72:273-80.

\section{Publisher's Note}

Springer Nature remains neutral with regard to jurisdictional claims in published maps and institutional affiliations.
Ready to submit your research? Choose BMC and benefit from:

- fast, convenient online submission

- thorough peer review by experienced researchers in your field

- rapid publication on acceptance

- support for research data, including large and complex data types

- gold Open Access which fosters wider collaboration and increased citations

- maximum visibility for your research: over 100M website views per year

At BMC, research is always in progress.

Learn more biomedcentral.com/submissions 